\title{
PROOF OF SOME ASYMPTOTIC RESULTS FOR A MODEL EQUATION FOR LOW REYNOLDS NUMBER FLOW*
}

\author{
D. S. COHEN, $\dagger$ A. FOKAS $\dagger$ AND P. A. LAGERSTROM $\dagger$
}

\begin{abstract}
A two-point boundary value problem in the interval $[\varepsilon, \infty], \varepsilon>0$ is studied. The problem contains additional parameters $\alpha \geqq 0, \beta \geqq 0,0 \leqq U<\infty, k=$ real. It was originally proposed by Lagerstrom as a model for viscous flow at low Reynolds numbers. A related initial value problem is transformed into an integral equation which is shown to have a unique solution by a pincer method. The integral representation is used for a simple proof of the existence of a solution of the boundary value problem for $\alpha>0$; for $\alpha=0$ an explicit construction shows that no solution exists unless $k>1$. A special method is used to show uniqueness. For $\varepsilon \downarrow 0, k \geqq 1$, various results had previously been obtained by the method of matched asymptotic expansions. Examples of these results are verified rigorously using the integral representation. For $k<1$, the problem is shown not to be a layer-type problem, a fact previously demonstrated explicitly for $k=0$. If $k$ is an integer $\geqq 0$ the intuitive understanding of the problem is aided by regarding it as spherically symmetric in $k+1$ dimensions. In the present study, however, $k$ may be any real number, even negative.
\end{abstract}

\section{Introduction. Basic problem and its asymptotic solutions.}

Formulation of problem and discussion of previous research. The following problem was introduced by one of the authors (Lagerstrom (1961))

$$
\begin{gathered}
\frac{d^{2} u}{d x^{2}}+\frac{k}{x} \frac{d u}{d x}+\alpha u \frac{d u}{d x}+\beta\left(\frac{d u}{d x}\right)^{2}=0, \\
u=0 \quad \text { at } x=\varepsilon>0, \quad u=U \geqq 0 \quad \text { at } x=\infty .
\end{gathered}
$$

The purpose was to provide an analytically relatively simple model to illustrate the ideas and techniques used by Saul Kaplun in his asymptotic treatment of flow past a solid at low Reynolds numbers (corresponding to $\varepsilon$ small). ${ }^{1}$

The intuitive thinking about (1.1) will be aided if one gives it the following, admittedly somewhat unrealistic physical interpretation. We regard $x$ as a radial coordinate in $(k+1)$-dimensional space and $u$ as temperature. Equation (1.1a) is then an equation for time-independent temperature-distribution in an infinite medium. The first two terms are Laplace's operator, the last two represent nonlinear autonomous heat sources (not occurring in any known actual physical problem!). The temperature is zero on the sphere $x=\varepsilon$ and $U$ at large distances. Using this intuitive interpretation, Lagerstrom (1961) applied Kaplun's ideas to construct asymptotic solutions for two $(k=1)$ and three $(k=2)$ dimensions, for $\beta=0$ and $\beta=1$. $^{2}$ The intuitive reasoning indicated that for one dimension $(k=0)$ the problem is not singular. This was verified by the construction of an exact solution for this case. If $\alpha>0$, or $\beta>0$ one may make their values unity (even simultaneously) by a scale transformation in $u$ and $x$. However since $\beta=0$ is an interesting case and $\alpha=0$ occurs

* Received by the editors August 29, 1977. Original research in 1966-67 was supported in part by the U.S. Air Force under Contract AF-AFOSR-338-65. Recent research supported in part by the National Science Foundation under Grant MCS 75-05414.

† Department of Applied Mathematics, California Institute of Technology, Pasadena, California 91125.

${ }^{1}$ Knowledge of fluid dynamics will not be necessary for reading this paper even though we shall use terms from that subject, such as "Stokes equation," "Oseen equation," etc. For those familiar with the corresponding problems in fluid dynamics such terms will help to establish the connection; others may simply regard them as distinctive names.

${ }^{2}$ Asymptotic results were also obtained for $\varepsilon$ very large. This problem however, does not correspond to flow at large Reynolds numbers, and will not be discussed in the present paper. 
in an auxiliary equation (the Stokes equation), we shall consider arbitrary nonnegative values of $\alpha$ and $\beta$. We shall assume $\varepsilon>0$ and, since this does not introduce any mathematical complications, let $k$ be any real number. For $\alpha>0,(1.1)$ has a unique solution. Singular asymptotic techniques may, however, be used only if $k \geqq 1$. For $\alpha=0$, (1.1a) may be solved explicitly. The boundary value problem then has a solution (which is unique) only if $k>1$. A corresponding initial-value problem may be solved explicitly for any value of $k$. Thus $k=1$ is an important limiting case.

The original report (Lagerstrom (1961)) was (and is) not easily available. The asymptotic ideas are briefly discussed in Lagerstrom's induction to Part I of the posthumous edition of Kaplun's collected works (1967). Some previously obtained results are given in Cole's book (1968), for the case $\beta=0$. The asymptotic analysis was carried further by Bush (1971), Lagerstrom-Casten (1972), and Lagerstrom (1976).

Ideas for a rigorous discussion of (1.1) were given, in rather sketchy form, by Cole (1968). He assumed $\beta=0$ and utilized an invariance group of $(1.1 \mathrm{a})$, which is destroyed when $\beta>0$. Hsiao (1973) gave a rigorous discussion of the existence of a solution of (1.1) for $\beta=0, k=1$, and $\varepsilon \downarrow 0$. He proved the validity of some asymptotic results found earlier. In particular he gave the general form of the part of the asymptotic expansion which neglects transcendentally small terms (such terms will not be discussed here). Recently MacGillivray (1977) has obtained rigorous results for $k=2, \beta=1$. In the present paper there are few restrictions on the values of the parameters. The restrictions are mainly necessitated by the fact that (1.1) must have a solution. The integral equation introduced in $\S 2$ is similar to that used by Cole (1968); however, the group invariance of (1.1a) used by Cole is known only for $\beta=0$ and our use of the integral equation is quite different from that of Cole's. Our methods differ radically from those used by Hsiao and MacGillivray and may possibly be more powerful. However, Hsiao's method, restricted to $k=1$ and $\beta=0$, may have the advantage of being applicable to the "real" problem, i.e., to the problem in fluid dynamics for which (1.1) is a model.

\section{Review of some simple asymptotic results.}

1) Outer Solution. The first principal limit for $k \geqq 1$ is $\varepsilon \downarrow 0, x$ fixed. This gives the first term of the outer expansion

$$
f_{0}=U \text {. }
$$

2) Inner Solution. Since (1.2) does not give an approximation valid at near $x=\varepsilon$ one introduces an inner variable

$$
\tilde{x}=x \varepsilon^{-1}
$$

and, $g_{0}$, the first term of the inner solution satisfies the Stokes equation

$$
\frac{d^{2} g_{0}}{d \tilde{x}^{2}}+\frac{k}{\tilde{x}} \frac{d g_{0}}{d \tilde{x}}+\beta\left(\frac{d g_{0}}{d \tilde{x}}\right)^{2}=0
$$

and the inner boundary condition, $g_{0}=0$ at $x=\varepsilon, \tilde{x}=1$. The solution $w$ of (1.4) satisfying the initial conditions

$$
w=A, \quad \frac{d w}{d \tilde{x}}=B \quad \text { at } \tilde{x}=1,
$$

is

$$
\beta=0 ; k=1 \text { : }
$$

$$
w=B \ln \tilde{x}+A
$$


$\beta=0 ; k \neq 1$ :

$$
w=\frac{B \tilde{x}^{1-k}}{1-k}+A-\frac{B}{1-k}
$$

$\beta=1 ; k=1$ :

$$
w=A+\ln (B \ln \tilde{x}+1)
$$

$\beta=1 ; k \neq 1$ :

$$
w=A+\ln \frac{B \tilde{x}^{1-k}+1-k-B}{1-k} .
$$

(The solutions for $\beta=0$ are trivial; those for $\beta=1$ are obtained using the simple observation that if $w$ satisfies (1.4) with $\beta=1$ then $e^{w}$ satisfies (1.4) with $\beta=0$.) For $k \leqq 1$ the boundary conditions $\tilde{g}(1)=0, g_{0}(\infty)=U \neq 0$ can never be satisfied. For $k=1$ this corresponds to the Stokes paradox (see result 6) below).

3) Nonlinearity of the general Stokes equation. Contrary to what used to be stated in the hydrodynamical literature, the Stokes equation is not obtained by linearizing around $u=0$ but by a limit process applied to the full equation. For $\beta=0$ (corresponding to incompressible flow) it is linear by accident but for $\beta=1$ (corresponding to compressible flow) it is nonlinear.

4) Oseen equation. The outer expansion starts, for $k \geqq 1$,

$$
\begin{gathered}
u \sim f_{0}+C_{1} \zeta(\varepsilon) f_{1}+\cdots, \zeta(\varepsilon)=o(1), \\
f_{0} \equiv U .
\end{gathered}
$$

Thus $f_{1}$ obeys an Oseen equation which may be obtained by linearizing around the value at infinity. If we assume $U=1$, the Oseen equation is

$$
\frac{d^{2} f_{1}}{d x^{2}}+\frac{k}{x} \frac{d f_{1}}{d x}+\frac{d f_{1}}{d x}=0
$$

Thus the Oseen equation is by nature linear. For $\beta=0$ it contains the Stokes equation (the inner limit of (1.1a) is the same as that of (1.8).) This is, however, not the case for $\beta=1$. Thus for $\beta=0$ and $k>1,(1.8)$ has a solution which is uniformly valid to a certain order, that is, one may solve a two-point boundary value problem using (1.8) and satisfy the condition at $x=\varepsilon$ to a certain order (or even exactly; however, (1.8) is only approximate). For $k=1$ this corresponds to the Lamb solution in fluid mechanics. Again this is accidental; for $\beta=1$ the statement just made is no longer true. One may of course satisfy the boundary condition at $x=\varepsilon$ using (1.8) but the result is not a valid approximation for $\beta=1$.

The solution of (1.8) which vanishes at infinity is within a multiplicative constant the exponential integral

$$
E_{k}(x)=\int_{x}^{\infty} s^{-k} e^{-s} d s
$$

5) Special case $k=0$. In this case the problem is not a singular perturbation problem. This is obvious from the intuitive interpretation. In fact the exact solution for $\alpha=1, \beta=0$ is

$$
u=U \tanh \frac{U}{2}(x-\varepsilon)
$$


For $\alpha=1, \beta=1$ one finds, putting $p=d u / d x$, that either $p \equiv 0$ which corresponds to $U=0, u \equiv 0$ or

$$
p=A e^{-u}+(1-u) \text {. }
$$

Since $p$ tends to zero as $u \rightarrow \infty$ (this will follow from the results of $\S \S 2$ and 3 ) the constant of integration is

$$
A=e^{U}(U-1)
$$

Thus in principle the problem is solvable by quadrature and inversion of a function. In the special case $U=1$ one finds explicitly

$$
u=1-e^{-(x-\varepsilon)} \text {. }
$$

Note that $x$ and $\varepsilon$ occur only in the combination $(x-\varepsilon)$. This is due to the invariance of the equations under the group $x \rightarrow x+a$ and shows the nonsingular behavior of the solution. The special dependence of (1.10) on $U$ is due to invariance under the group $x \rightarrow a x, u \rightarrow a^{-1} x$. A corresponding group for the case $\beta=1$ is not known.

6) Note on the dependence on $k$. We shall consider all real values of $k$ including negative values. For $k \geqq 1$ and integer, intuitive reasoning tells us that the problem is singular and that $f_{0}=U$. Since the "cooling power" of the "sphere" $x=\varepsilon$ decreases with increasing integer $k$, it is reasonable to assume that the same is true for any value $k \geqq 1$, so that $f_{0}=U$. For $k<1$ an intuitive interpretation is difficult except for the case $k=0$ discussed above. From (1.6) one sees that for $k \leqq 1$ the boundary condition at infinity cannot be satisfied by a solution of (1.4). However, for $k=1$ matching with $f_{0}=U$ is still possible, ${ }^{3}$ whereas for $k<1$ such a matching is not possible. It is therefore natural to assume that the problem is not singular for $k<1$ (or at least that the asymptotic techniques used for $k \geqq 1$ are not valid for $k<1$ ) and that the outer limit is not equal to $U$. It will however be shown that boundary-value problem (1.1) has a solution for any value $k$ if $\alpha>0$. In fact, it will be shown that if $M^{*}$ is the exact value of $(d u / d x)_{x=\varepsilon}$, the exact order of $M^{*}$ is $\varepsilon^{-k}$ for $k<1$, and is $\varepsilon^{-1}$ for $k>1$. The case $k=1$ is an important limiting case and the order of $M^{*}$ is $\varepsilon^{-1} \nu(\varepsilon)$ where $\nu(\varepsilon)={ }_{\mathrm{df}}|\ln \varepsilon|^{-1}$.

7) First term in inner expansion. This term will be denoted by $g_{0}(\tilde{x})$. It must vanish at $\tilde{x}=1$ and match with $f_{0}=1$. Denoting $d g_{0} / d \tilde{x}$ at $\tilde{x}=1$ by $\tilde{M}_{0}=\varepsilon M_{0}$ one finds

$$
\beta=0 ; k=1 \text { : }
$$

$$
g_{0}=\tilde{M}_{0} \ln \tilde{x}, \quad \tilde{M}_{0}=|\ln \varepsilon|^{-1}=\nu(\varepsilon)
$$

$\beta=0, k>1$ :

$$
g_{0}=\left(1-\frac{\tilde{M}_{0}}{k-1} \tilde{x}^{1-k}\right), \quad \tilde{M}_{0}=k-1
$$

$\beta=1, k=1$ :

$$
g_{0}=\ln \left[1+\tilde{M}_{0} \ln x\right], \quad \tilde{M}_{0}=(e-1) \nu(\varepsilon)
$$

$\beta=1 ; k>1$ :

$$
g_{0}=\ln \left[e-\frac{\tilde{M}_{0}}{k-1} \tilde{x}^{1-k}\right], \quad \tilde{M}_{0}=(e-1)(k-1)
$$

\footnotetext{
${ }^{3}$ This is, in essence, Kaplun's resolution of the Stokes paradox.
} 
Thus for $k>1, g_{0}$ satisfies the boundary condition at infinity. However, if one rewrites (1.1a) in $\tilde{x}$ variables and treats it as a regular perturbation problem, some higher order term will tend to infinity with $\tilde{x}$. For $k=2$ it occurs in the second term; this is known as Whitehead's paradox. As $k$ increases the infinity will occur in higher-order terms. The "paradox" is thus delayed. It will, however, always occur sooner or later; it is resolved by matching.

8) First two terms of outer expansion. By matching with $g_{0}(\tilde{x})$ one finds the second term of $(1.7 \mathrm{a})$. The results are $\left(E_{k}(x)\right.$ is defined by (1.9))

$\beta=0, k=1$ :

$$
f_{0}+C_{1} \zeta(\varepsilon) f_{1}=1-\nu(\varepsilon) E_{1}(x), \quad \nu(\varepsilon)=|\ln \varepsilon|^{-1} ;
$$

$\beta=0, k>1$ :

$$
f_{0}+C_{1} \zeta(\varepsilon) f_{1}=1-(k-1) \varepsilon^{k-1} E_{k}(x)
$$

$\beta=1, k=1$ :

$$
f_{0}+C_{1} \zeta(\varepsilon) f_{1}(x)=1-\frac{e-1}{e} \nu(\varepsilon) E_{1}(x)
$$

$\beta=1, k>1$ :

$$
f_{0}+C_{1} \zeta(\varepsilon) f_{1}(x)=1-\frac{(k-1)(e-1)}{e} \varepsilon^{k-1} E_{k}(x) .
$$

The above results are those of Lagerstrom (1961), trivially generalized to noninteger $k$, with the exception of (1.11)-(1.13) which were found recently by Fokas. Asymptotic results to higher order are given in the references cited above.

Historical note. Kaplun's original discoveries were published in 1957; see Kaplun-Lagerstrom (1957) and Kaplun (1957), reprinted in Kaplun (1967). Possibly, because these publications were in rather brief and condensed form, or possibly because of the novelty and of the ideas used they were mostly ignored or not understood. ${ }^{4}$ It was therefore felt that a rigorous analysis might help to persuade the doubters. The original work on the present paper was done a considerable time ago by D. S. Cohen and P. A. Lagerstrom. A preliminary draft was written early in 1967 and even referred to as a forthcoming publication. For various reasons, the authors were side-tracked by other problems. Also, while the uniqueness of a corresponding initial value problem was easily proved, only the asymptotic uniqueness of the boundary value problem was proved. Although the authors were more interested in construction based on intuitive arguments than in rigorous proofs they eventually decided to live up to their promise of a publication. A third author, A. Fokas, was enlisted and made several important contributions, in particular, regarding the uniqueness of the boundary value problem for any $\varepsilon>0$ (see $\S 3$ ).

Outline of the paper. In $\S 2$ an initial value problem is studied: The boundary condition at infinity is replaced by the condition $d u / d x=M$ at $x=\varepsilon$. The problem may then be recast in the form of an integral equation, very similar to the integral

${ }^{4}$ An important exception is the paper by Proudman-Pearson (1957). Basing their analysis on a short partial account of Kaplun's ideas published in Lagerstrom-Cole (1955, p. 873ff) and their independent analysis of the problem, Proudman and Pearson (1957) discussed the low-Reynolds number problem, discovering some significant features of the three-dimensional case. These features were shown to have their analogue in the model problem (1.1). A discussion of this is given in various references, the most complete is found in Lagerstrom-Casten (1972). 
equation which Weyl (1942) derived from the Blasius equation. The method of solution is also similar to that of Weyl ("pincer movement"). Starting with $u_{0}=0$ and applying the integral operator repeatedly one finds one increasing and one decreasing sequence of functions, each of which converge to the same function $u$ which is thus a fixed point of the integral operator and a solution of the initial value problem. In $\S 3$ the existence and uniqueness of the solution of boundary value problems is proved. For the Blasius equation, which has a simple group, the initial value problem can easily be related to the boundary value problem. A corresponding group exists for $\beta=0$, see Cole $(1968$, p. $61 \mathrm{ff})$, but has not been found for the more interesting case $\beta=1$. Thus in the latter case we have to abandom Weyl's method and prove the following: As $M$ varies, $M \geqq 0$, the value of $u(\infty)$ can be any number in $[0, \infty)$. The problem is then, does it assume the value $u(\infty)=U$ only once? One of the authors (A. Fokas) proved that $\partial u / \partial M$ exists and is positive for $x>\varepsilon$ even at $x=\infty$. Thus the condition $u(\infty)=U$ is achieved only for a specific value of $M$. This proves the uniqueness of the original boundary value problem, for any $\varepsilon>0$. Finally, in $\S 4$, the validity of the asymptotic formulas given above (which were derived by nonrigorous singular perturbation techniques) will be established rigorously.

Notation and terminology. The general notations of singular perturbation techniques (such as domain of validity, overlap, etc.) are explained in Lagerstrom-Casten. We shall, however, use slight changes of notation: The symbol "«" will be replaced by " $<$ ",

$$
\begin{aligned}
& \zeta(\varepsilon)<\eta(\varepsilon), \text { or } \quad \eta(\varepsilon)>\zeta(\varepsilon) \Leftrightarrow \lim _{\varepsilon \downarrow 0} \frac{\zeta}{\eta}=0, \\
& \zeta(\varepsilon) \leq \eta(\varepsilon), \quad \text { or } \eta(\varepsilon) \geq \zeta(\varepsilon) \Leftrightarrow \lim _{\varepsilon \downarrow 0}\left|\frac{\zeta}{\eta}\right|<\infty, \\
& \zeta(\varepsilon) \leq \eta(\varepsilon) \text { and } \eta(\varepsilon) \geq \zeta(\varepsilon) \Leftrightarrow \zeta \frac{\cup}{\cap} \eta .
\end{aligned}
$$

The last definition means that $\zeta(\varepsilon)$ and $\eta(\varepsilon)$ are of the same order. One may also write $\eta=O(\zeta)$, or $\zeta=O(\eta)$. However, the notation of $(1.16 \mathrm{c})$ brings out the fact that "being of the same order as" is a symmetric relation; it is actually an equivalence relation.

2. The initial-value problem. In (1.1) we replace the boundary condition at infinity by an additional initial value. The problem is then

$$
\begin{gathered}
\frac{d^{2} u}{d x^{2}}+\frac{k}{x} \frac{d u}{d x}+\alpha u \frac{d u}{d x}+\beta\left(\frac{d u}{d x}\right)^{2}=0, \\
u(\varepsilon)=0, \quad\left(\frac{d u}{d x}\right)_{x=\varepsilon}=M \geqq 0 .
\end{gathered}
$$

We shall show that a unique solution exists for $\alpha, \beta, \geqq 0, \varepsilon>0, k=$ real number. Rewriting (2.1a) as

$$
\left(x^{k} u^{\prime}\right)^{\prime}+\left(\alpha u+\beta u^{\prime}\right)\left(x^{k} u^{\prime}\right)=0,
$$

and using an integrating factor one finds

$$
x^{k} u^{\prime} \exp [\alpha U(x)+\beta u(x)]=\varepsilon^{k} M,
$$


where

$$
U(x)=\int_{\varepsilon}^{x} u(t) d t
$$

Thus, for $M>0$,

$$
\begin{aligned}
& x \geqq \varepsilon \Rightarrow u^{\prime}(x)>0, \\
& x>\varepsilon \Rightarrow u(x)>0 .
\end{aligned}
$$

An equivalent integral equation. It follows from the above that one may now rewrite (2.1) $\mathrm{as}^{5}$

$$
u=T[u]
$$

where the integral operator $T$ is defined by

$$
\begin{gathered}
T[f]=\varepsilon^{k} M \int_{\varepsilon}^{x} s^{-k} \exp [-\beta f(s)-\alpha F(s)] d s, \\
F(s)=\int_{\varepsilon}^{s} f(t) d t .
\end{gathered}
$$

Given two continuous functions $f(x), g(x)$ defined for $x \geqq \varepsilon$ we shall use the following notation:

$$
f\left(x_{0}\right)<g\left(x_{0}\right) \text { means strict inequality for } x=x_{0},
$$

(2.6b) $f<g$ means $f(x) \leqq g(x)$ for all $x$ and $f(x)<g(x)$ for $x$ in some finite interval.

Thus, provided the integrals exist and $M>0$,

$$
\begin{gathered}
T[f]>0, \\
f<g \Rightarrow T[g]<T[f] .
\end{gathered}
$$

Pincer movement. Following Weyl (1942) we show that (2.4) has a unique solution by using a pincer movement. We define

$$
u_{0}=0, \quad u_{n+1}=T\left[u_{n}\right] .
$$

If $M=0$, then $u_{n}=u_{0} \quad 0$, all $n$. Hence we assume $M>0$. Then by (2.7), $u_{1}>0$, $0<u_{2}<u_{1}$, from which une finds by an easy induction proof

$$
u_{0}<u_{2}<\cdots u_{2 l}<u_{2 l+1} \cdots u_{3}<u_{1} .
$$

Note that the functions $u_{m}$ are defined and finite for all $\varepsilon \leqq x \leqq \infty$ and belong to $C_{\infty}$. Obviously the sequences $\left(u_{2 l}\right)$ and $\left(u_{2 l+1}\right)$ converge pointwise to functions $u_{\mathrm{I}}$ and $u_{\mathrm{II}}$ respectively, with $u_{\mathrm{I}}(x) \leqq u_{\mathrm{II}}(x)$. To show the existence of a solution of (2.1), or equivalently a fixed point of the operator $T$, one needs to show strict equality

$$
u_{\mathrm{I}}(x)=u_{\mathrm{II}}(x) \text {. }
$$

First a few preliminary remarks. It is often convenient to use $\tilde{x}=x \varepsilon^{-1}$; the variable $\tilde{x}$ also occurs in the asymptotic analysis. Strictly speaking, corresponding to a

\footnotetext{
${ }^{5}$ As will be seen in $\S 4$, this integral equation (or integral representation of $u$ in terms of itself) will not only serve to solve the initial value problem; crude estimmates of $u$, based directly on (1.1) can give more useful estimates of $u$ with the aid of (2.4).
} 
function $f(x, \varepsilon)$ one should use different notation when $\tilde{x}$ is used, e.g., $\tilde{g}(\tilde{x}, \varepsilon)=f(x, \varepsilon)$. We shall, however, use $f$ instead of $g$ when there is no danger of ambiguity. The operator (2.5a) may then be written

$$
T[f]=\tilde{M} \int_{1}^{\tilde{x}} s^{-k} \exp \left[-\beta f(s)-\alpha \varepsilon \int_{1}^{s} f(t) d t\right] d s,
$$

where

$$
\tilde{M}=\varepsilon M=\left(\frac{d f}{d \tilde{x}}\right)_{\tilde{x}=1}
$$

The formulas for $u_{1}$ are

$$
\begin{aligned}
& u_{1}=\varepsilon^{k} M \frac{x^{1-k}-\varepsilon^{1-k}}{1-k}=\tilde{M} \frac{\tilde{x}^{1-k}-1}{1-k}, \quad k \neq 1, \\
& u_{1}=\varepsilon M(\ln x-\ln \varepsilon)=\tilde{M} \ln \tilde{x}, \quad k=1 .
\end{aligned}
$$

To prove (2.10) we need the following

LEMMA 2.1. If $\alpha \geqq 0, \beta \geqq 0, M \geqq 0, \varepsilon>0$ then

$$
\left|u_{m}-u_{m+1}\right| \rightarrow 0 \text { as } m \rightarrow \infty \text {. }
$$

Proof. If $\alpha=\beta=0$ then $u_{1}=u_{m}$ for $m \geqq 1$, where $u_{1}$ is given by (2.12). In fact, existence of a solution for the boundary value problem for $k>1$ follows from (2.12). We may then assume $\alpha+\beta>0$ and make a positive scale transformation of $u$ such that

(a)

$$
\varepsilon \alpha+\beta=2 .
$$

This scale transformation may change the values of $M$ and of $\varepsilon$; this is irrelevant since they are arbitrary positive numbers.

From $0 \leqq u \leqq v \Rightarrow e^{-u}-e^{-v} \leqq v-u$ we derive a useful estimate

$$
\begin{aligned}
\left|u_{m+1}(x)-u_{m}(x)\right|<\tilde{M} \int_{1}^{x} s^{-k}\{ & {\left[\varepsilon \alpha \int_{1}^{s}\left|u_{m}(t)-u_{m-1}(t)\right| d t\right] } \\
& \left.+\beta\left|u_{m}(s)-u_{m-1}(s)\right|\right\} d s .
\end{aligned}
$$

We shall give various upper bounds for $\left|u_{n}-u_{n-1}\right|$ for various ranges of $k$, and prove them by induction. In all cases the estimates are directly verified for $n=1$, by use of (2.12); hence in the induction proof we need only consider the step from $n$ to $n+1$.

For $k<1$,

$$
\left|u_{m}-u_{m-1}\right| \leqq \frac{(2 \tilde{M})^{m}(\tilde{x})^{(1-k) m}(\tilde{x})^{2 m}}{(1-k) m !}
$$

Integrating the induction hypothesis one finds

$$
\int_{1}^{s}\left|u_{m}(t)-u_{m-1}(t)\right| d t \leqq \frac{(2 \tilde{M})^{m} s^{(1-k) m}}{(1-k) m !} \cdot \frac{s^{2 m+1}}{2 m+1} .
$$

Applying the integral operator $T$ to (c) gives

$$
\text { (e) }\left|u_{m+1}-u_{m}\right| \leqslant \frac{\tilde{M}(2 \tilde{M})^{m} \tilde{x}^{(1-k)(m+1)}}{(1-k) m !}\left[\int_{1}^{\tilde{x}} \frac{1}{s}\left[\varepsilon \alpha^{2} \frac{s^{m+1}}{2 m+1}+\beta s^{m}\right] d s\right] \text {. }
$$


In this estimate it is essential that, since $k<1$, in the integral $\int_{1}^{\tilde{x}} s^{1-k}(\cdots) d s$ the factor $s^{1-k}$ takes on its largest value at the upper limit. Since

$$
\begin{aligned}
\int_{1}^{\tilde{x}} \frac{1}{s}\left(\varepsilon \alpha \frac{s^{2 m+1}}{2 m+1}+\beta s^{m}\right) d s & \leqq \frac{\varepsilon \alpha \tilde{x}^{2 m+1}}{(2 m+1)^{2}}+\beta \frac{\tilde{x}^{2 m}}{2 m} \\
& \leqq \varepsilon \alpha \frac{\tilde{x}^{2 m+2}}{(m+1)}+\beta \frac{\tilde{x}^{2 m+2}}{m+1}
\end{aligned}
$$

and $\varepsilon \alpha+\beta=2$, the induction is complete.

For $k=1$,

$$
\left|u_{m}-u_{m-1}\right| \leqq(2 \tilde{M})^{m} \ln \tilde{x} \frac{\tilde{x}^{2 m}}{m !} .
$$

Integrating the induction hypothesis one finds:

(g)

$$
\int_{1}^{s}\left|u_{m}(t)-u_{m-1}(t)\right| d t \leqq(2 \tilde{M})^{m} \frac{\ln s}{m !} \frac{s^{2 m+1}}{2 m+1} .
$$

Substituting (f) and (g) into (b) and evaluating the log term at the upper limit (where it assumes its largest value) we get:

This completes the induction.

$$
\begin{aligned}
\left|u_{m+1}-u_{m}\right| \leqq \frac{\tilde{M}(2 \tilde{M})^{m} \ln \tilde{x}}{m !} \int_{1}^{\tilde{x}} \frac{1}{s}\left[\frac{\varepsilon \alpha^{2 m+1}}{2 m+1}+\beta s^{2 m}\right] d s \\
\leqq \frac{\tilde{M}(2 \tilde{M})^{m} \ln \tilde{x}}{m !} \frac{2 \tilde{x}^{2(m+1)}}{m+1}
\end{aligned}
$$

For $k>1$,

$$
\left|u_{m}-u_{m-1}\right| \leqq \frac{(2 \tilde{M})^{m}}{k-1} \frac{\tilde{x}^{2 m}}{m !}
$$

Integrating the induction hypothesis one finds:

$$
\int_{1}^{s}\left|u_{m}(t)-u_{m-1}(t)\right| d t \leqq \frac{(2 \tilde{M})^{m}}{(k-1) m !} \frac{s^{2 m+1}}{2 m+1}
$$

substituting (h) and (e) into (b) and evaluating $1 / s^{k-1}$ at the lower limit where it takes its largest value we get

$$
\begin{aligned}
\left|u_{n+1}-u_{n}\right| & \leqq \frac{\tilde{M}(2 \tilde{M})^{m}}{(k-1) m !} \int_{1}^{\tilde{x}} \frac{1}{s}\left[\frac{\varepsilon \alpha s^{2 m+1}}{2 m+1}+\beta s^{2 m}\right] d s \\
& \leqq \frac{\tilde{M}(2 \tilde{M})^{m}}{(k-1) m !} \cdot \frac{2 \tilde{x}^{2(m+1)}}{m+1} .
\end{aligned}
$$

This completes the induction and the proof of Lemma 2.1 .

THEOREM 2.1. The initial value problem has a unique solution $u$.

Proof. Lemma 2.1 proves (2.10). Hence the pincer closes. More explicitly, writing

$$
u_{m}=\sum_{j=0}^{m-1}\left(u_{j+1}-u_{j}\right)
$$

and letting $m \rightarrow \infty$ one sees that from (c), (e) and (h) in the proof of the Lemma that the series $\sum\left|u_{j+1}-u_{j}\right|$ is majorized by an exponential series of some power of $x$. Thus, 
as $m \rightarrow \infty$, the series ( $\mathrm{j}$ ) is absolutely convergent to a function $u$ which is analytic in $x$.

Furthermore this solution is unique. If $\bar{u}$ is a solution then $0=u_{0}<\bar{u}$. Hence $T[\bar{u}]<u_{1}=T\left[u_{0}\right]$. As above one then proves $u_{2 m}<\bar{u}<u_{2 m+1}$. Since the pincer closes it follows that $\bar{u}=\lim u_{m}$ and hence $\bar{u}$ is identical with the solution found by a pincer movement.

3. The boundary-value problem. Existence and uniqueness of a solution of (1.1) will be shown for $\alpha>0$, or $\alpha=0$ and $k>1$. Explicit construction (see (1.12) and (1.13)) shows that if $\alpha=0, k \leqq 1$, the solution cannot take on a finite value at infinity.

Existence follows if one can show that by varying $M$ in the initial-value problem one can make $u(\infty)$ be any nonnegative number. To prove uniqueness one must show that $u(\infty)$ assumes each value only for one value of $M$. This will follow from a proof of the intuitively plausible assumption that the derivative of $u(\infty)$ with respect to $M$ is positive, even at $x=\infty$.

LemMA 3.1. Assume $M \geqq 0, \varepsilon>0$, or $\alpha=0$ and $k>1, \beta \geqq 0$. Then there exists a number $C$, independent of $x$, such that $u(x)<C$ for $\varepsilon \leqq x \leqq \infty$.

Proof. Let $\bar{x}$ be a fixed value of $x, \bar{x}>\varepsilon$. Define $\bar{u}=u(\bar{x})$ and a function $f(x)$ by

$$
\begin{array}{ll}
f=0, & \varepsilon \leqq x \leqq \bar{x}, \\
f=\bar{u}, & \bar{x}<x .
\end{array}
$$

Then $f<u$ and hence

(a)

$$
\begin{aligned}
u(x)=T[u]<T[f] & =\varepsilon^{k} M \int_{\varepsilon}^{\bar{x}} s^{-k} d s+\varepsilon^{k} M \int_{\bar{x}}^{x} s^{-k} e^{-\beta \bar{u}-\alpha \bar{u}(s-\bar{x})} d s \\
& <\varepsilon^{k} M \int_{\varepsilon}^{\bar{x}} s^{-k^{k}} d s+\varepsilon^{k} M \int_{\bar{x}}^{\infty} s^{-k} e^{-\beta \bar{u}-\alpha \bar{u}(s-\bar{x})} d s=I_{1}+I_{2} .
\end{aligned}
$$

Since $\bar{x}$ is fixed the integral $I_{1}$ is a fixed number. The improper integral $I_{2}$ exists under the assumptions of the lemma. Thus $I_{1}+I_{2}$ is a finite number independent of $x$ which may be taken as the constant $C$ of the lemma.

That the restriction of $\alpha$ and $k$ cannot be removed by a finer estimate follows from (1.14) and the uniqueness of the initial-value problem. If $\alpha=0, k \leqq 1$ then $u(\infty)=\infty$.

LEMMA 3.2. For fixed $\varepsilon>0, k, \alpha \geqq 0, \beta \geqq 0$, one may choose an $M$ such that $u(\infty)$ is greater than any given constant.

Proof. Take a fixed value $x=\bar{x}>\varepsilon$ and assume there is a constant $C$ such that $u(\bar{x})<C$, all $M$. Then

$$
u(x)<C, \quad \varepsilon \leqq x \leqq \bar{x}
$$

Hence

$$
T[C]_{x=\bar{x}}=\varepsilon^{k} M \int_{\varepsilon}^{\bar{x}} s^{-k} \exp [-[\alpha C(s-\varepsilon)-\beta C]] d s>u(\bar{x})
$$

Since $\varepsilon>0$ one may increase $M$ such that $T[C]_{x=\bar{x}}$ becomes greater than $C$. This contradicts the assumption $u(\bar{x})<C$. A fortiori, the value of $u(\infty)$ may be made arbitrarily large. 
LemMA 3.3. The function $u(x)=u(x ; M, \varepsilon, \alpha, \beta)$ is continuous and differentiable in $M$ in the interval $\varepsilon \leqq x<\infty$. Furthermore, if

$$
v=\frac{\partial u}{\partial M}
$$

then $v^{\prime}$ exists in the same interval.

Proof. The lemma follows from the uniqueness of the initial-value problem; see for instance Hartman (1964, p. 94ff).

In order to extend Lemma 3.3 to $x=\infty$ we need to show that $v(x)$ is nonnegative and bounded.

LeMma 3.4. $v(x)>0$ in $(\varepsilon, \infty)$.

Proof. Define

$$
\begin{aligned}
u_{M}(x)= & \text { the unique solution of the } \\
& \text { initial-value problem for } u(x) \text { with } u^{\prime}(\varepsilon)=M,
\end{aligned}
$$

and consider it as a given function. It is then the solution of the linear initial-value problem in $u$,

$$
\begin{aligned}
& u^{\prime \prime}+\left(\frac{k}{x}+\beta u_{M}^{\prime}\right) u^{\prime}+\alpha u_{M}^{\prime} u=0, \\
& u=0, \quad u^{\prime}=M \quad \text { at } x=\varepsilon .
\end{aligned}
$$

This function will be compared with $v$ which is the solution of the linear problem

$$
\begin{gathered}
v^{\prime \prime}+\left(\frac{k}{x}+\alpha u_{M}+2 \beta u_{M}^{\prime}\right) v^{\prime}+\alpha u_{M}^{\prime} v=0, \\
v=0, \quad v^{\prime}=1 \quad \text { at } x=\varepsilon .
\end{gathered}
$$

Comparing (3.3a) and (3.4a) one sees that the coefficients of $u$ and $v$ are the same, the coefficient of $u^{\prime}$ is

$$
g_{2}=\frac{k}{x}+\beta u_{M}^{\prime}
$$

and that of $v^{\prime}$ is

$$
g_{1}=g_{2}+\alpha u_{M}+\beta u_{M}^{\prime}
$$

A straightforward calculation gives

$$
g_{1}^{\prime}+\frac{1}{2} g_{1}^{2}=g_{2}^{\prime}+\frac{1}{2} g_{2}^{2}+\frac{1}{2}\left(\alpha u_{M}+\beta u_{M}^{\prime}\right)^{2}+\alpha\left(\frac{k}{x} u_{M}+u_{M}^{\prime}\right)
$$

Since $u_{M}(\varepsilon)=0, u_{M}(x)>0$ for $x>\varepsilon, u_{M}^{\prime}(x)>0$ and since $v>0$ at least in some open interval with $\varepsilon$ as a left endpoint one concludes from (c) and Theorem 20 of ProtterWeinberger (1967, pp. 45-46) that $v$ can have at most one zero in any closed finite interval $\left[\varepsilon, x_{0}\right]$. Since $v(\varepsilon)=0$ the lemma follows.

LEMMA 3.5. $v(x) \leqq u_{M}(\infty) / M$ in $\varepsilon \leqq x<\infty$.

Proof. Differentiating the integral equation (2.4) with respect to $M$ one finds

$$
v(x)=\frac{u_{M}(x)}{M}-\varepsilon^{k} M \int_{\varepsilon}^{x} s^{-k}(\alpha V(s)+\beta v(x)) e^{-\alpha U_{M}(s)-\beta u_{M}(s)} d s,
$$


where

$$
V(s)=\int_{\varepsilon}^{s} v(t) d t, \quad U_{M}(s)=\int_{\varepsilon}^{s} u_{M}(t) d t .
$$

Since $v(x) \geqq 0, \alpha, \beta \geqq 0$ and $u_{M}(x) \leqq u_{M}(\infty)$ the lemma follows.

Discussion. The bound on $v$ as given in the lemma is not defined for $M=0$. If $M=0$ then

$$
u \equiv 0,
$$

and

$$
v(x)=\varepsilon^{k} \int_{\varepsilon}^{x} s^{-k} d s
$$

Thus for $M=0$ and $k \leqq 1, v(\infty)=\infty$. However, we shall only need the fact that $u(\infty, M)$ is continuous from the right in $M$ at $M=0$. This will be proved later (Lemma 3.8). To illustrate the situation consider the explicit formula (1.10) for the case $k=0$, $\alpha=1, \beta=0$. In this case $M=U^{2} / 2, u(\infty)=U=\sqrt{2 M}, v(\infty)=1 / U=1 / \sqrt{2 M}$. At $M=0, v=\infty$, but $\lim _{M \downarrow 0} u(\infty, M)=0=u(\infty, 0)$.

LEMMA 3.6. If $\alpha>0$, or $\alpha=0$ and $k>1$, and $M>0$ the limit $v(\infty)=\lim _{x \rightarrow \infty} v(x)$ exists and is finite. Furthermore,

$$
v(\infty)=\frac{\partial u_{M}(\infty)}{\partial M}
$$

Proof. By Lemmas 3.4 and 3.5

$$
0 \leqq V(s) \leqq \frac{u_{M}(\infty)(s-\varepsilon)}{M},
$$

and for $s \geqq s_{0}>\varepsilon$

(b)

$$
U_{M}(s) \geqq u_{M}\left(s_{0}\right)(s-\varepsilon) .
$$

Using the estimates (a) and (b) one sees from (3.5) that the improper integral $(x=\infty)$, defining $v(\infty)$, exists under the assumptions of the lemma. This integral is uniformly convergent in $M$ which proves (3.7).

LEMMA 3.7. $v(\infty)>0$.

Proof. In view of Lemma 3.4 we need only to prove that $v(\infty) \neq 0$. Rewriting (3.4a) as

$$
v^{\prime \prime}+\left(\frac{k}{x}+2 \beta u_{M}^{\prime}\right) v^{\prime}+\alpha\left(u_{M} v\right)^{\prime}=0
$$

one finds by integration and using $(3.4 \mathrm{~b}, \mathrm{c})$,

$$
v^{\prime}+\int_{\varepsilon}^{x}\left[k s^{-1}+2 \beta u_{M}^{\prime}(s)\right] v^{\prime}(s) d s=1
$$

Since $u_{M}(x)$ is increasing with $x$ and tends to a finite value as $x \rightarrow \infty$ it follows that

$$
u_{M}^{\prime}(\infty)=0 \text {, }
$$

and hence

$$
v^{\prime}(\infty)=0 .
$$


Assume now

(e)

$$
v(\infty)=0 .
$$

Then from (b) and (d)

$$
1-\int_{\varepsilon}^{\infty}\left[k s^{-1}+2 \beta u_{M}^{\prime}(s)\right] v^{\prime}(s) d s<\left(\frac{|k|}{\varepsilon}+2 \beta M\right) v(\infty)
$$

Thus the impossibility of (e) follows from (f).

LEMMA 3.8. $u(\infty, M)$ is continuous for $M \geqq 0$.

Proof. For $M>0$ continuity follows from differentiability. Since $u(\infty, 0)=0$ we need to prove

$$
\lim _{M \downarrow 0} u(\infty, M)=0 .
$$

(This one-sided continuity is all which is needed for the next theorem.) Assume that the limit in (a) is not zero but a constant $C>0$. Since $M>0$ implies $v(\infty)>0$ a consequence of this is

$$
u(\infty, M) \geqq C, \quad \text { all } M>0 .
$$

If (b) holds there must be an $\bar{x}$ such that

$$
u(\bar{x})=\frac{C}{2} .
$$

Here $\bar{x}$ might depend on $M$. However its least upper bound for $0<M \leqq$ const. cannot be infinite since this would contradict (b). Its least upper bound is then a finite constant $\bar{x}$ independent of $\varepsilon$. Thus the function which is zero in $\varepsilon \leqq x \leqq \bar{x}$ and is equal to $C / 2$ for $\bar{x} \leqq x$ is smaller than $u(x)$. We now replace $\bar{u}$ in formula (a) of the proof of Lemma 3.1 by $C / 2$. Then $M$ enters only as a multiplicative factor in the two integrals. Hence by letting $M \downarrow 0$ one may make $u(x)$ uniformly bounded by any number $>0$. Lemma 3.8 is thus proved by contradiction.

Discussion. It follows that $u(\infty, M)$ is continuous in $M$ and monotonely increasing, for $M \geqq 0$. It has not been proved that the derivative with respect to $M$ exists at $M=0$. As shown in the discussion of lemma $3.5, v(\infty, M)$ may actually tend to infinity as $M \downarrow 0$.

THEOREM $3.1 .^{6}$ If $\alpha>0$, or $\alpha=0$ and $k>1$, the boundary value problem (1.1) has a unique solution.

Proof. Existence follows from the fact that $u(\infty, M)$ is equal to 0 at $M=0$, can be made arbitrarily large by varying $M$ but is not infinite, and is continuous in $M$.

Uniqueness follows from the fact that $v(\infty)>0$ for $M>0$. It must be noted that $M<0$ can never give a nonnegative value of $u$ at $x=\infty$ (cf. (2.4) and (2.5)) and that $u \equiv 0$ is clearly the unique solution for $u(\infty)=0$.

\section{Proof of some asymptotic results.}

4.1. Introduction. Notation. Having established existence, uniqueness and certain properties of the exact solution of the boundary-value problem (1.1) we shall now prove rigorously various theorems about the asymptotic formulas of $\S 1$, which were obtained by singular-perturbation techniques. We shall restrict ourselves to

\footnotetext{
${ }^{6}$ The authors are indebted to A. D. MacGillivray for pointing out that a proof given in an earlier draft was incomplete.
} 
lower-order approximations. The problem is open-ended; one could in principle prove the validity of approximations to any order. This would not involve any essential new ideas for any partial approximation. Ideally, one should show that the method used gives valid approximations to any order. A solution of this much deeper problem will not be attempted here.

For $\alpha=0, k>1$, the solution can be given explicitly and for $\alpha=0, k \leqq 1$, no solution exists. Thus we shall assume $\alpha>0$. The case $\beta>0$ is more complicated than the case $\beta=0$. By a scale transformation one may make $\alpha=1$ and, if $\beta>0, \beta=1$ simultaneously. For $u(\infty)=0$, the problem has the unique solution $u \equiv 0$. For simplicity we assume $U=u(\infty)=1$; the ideas used below are the same for any strictly positive value of $u(\infty)$. The values to be used are thus

$$
\alpha=1, \quad \beta=0 \quad \text { or } \beta=1, \quad u(\infty)=1 \text {. }
$$

We shall first consider arbitrary real values of $k$, incidentally verifying that the asymptotic techniques used for $k \geqq 1$ are not applicable for $k<1$. After this, only the case $k \geqq 1$ will be considered.

The exact solution of (1.1) will be denoted by $u^{*}(x)$; it depends on the parameter $k$ and on the value of $\beta$. We define

$$
U^{*}(x)=\int_{\varepsilon}^{x} u^{*}(s) d s
$$

and

$$
M^{*}=\left(\frac{d u^{*}}{d x}\right)_{x=\varepsilon}, \quad \tilde{M}^{*}=\varepsilon M^{*} .
$$

The integral operator (2.5a) with $M=M^{*}$ will be denoted by $T^{*}$. Thus

$$
\begin{aligned}
u^{*}(x) & =T^{*}\left[u^{*}\right]=\varepsilon^{k} M^{*} \int_{\varepsilon}^{x} s^{-k} \exp \left[-\beta u^{*}(s)-U^{*}(s)\right] d s \\
& =\tilde{M}^{*} \int_{1}^{\tilde{x}} s^{-k} \exp \left[-\beta u^{*}(s)-\varepsilon \int_{1}^{s} u^{*}(t) d t\right] d s
\end{aligned}
$$

For the inner solution we shall need an operator with $\alpha=0$ (corresponding to the Stokes equation). It will be denoted by $T_{0}$ :

$$
\begin{aligned}
T_{0}[f] & =T_{0}[f ; M]=\varepsilon^{k} M \int_{\varepsilon}^{x} s^{-k} e^{-\beta f(s)} d s \\
& =\tilde{M} \int_{1}^{\tilde{x}} s^{-k} e^{-\beta f(s)} d s, \quad \tilde{M}=\varepsilon M .
\end{aligned}
$$

We define

$$
T_{0}^{*}[f]=T_{0}\left[f ; M^{*}\right]
$$

and its fixed point by $w^{*}$ :

$$
w^{*}=T_{0}^{*}\left[w^{*}\right] .
$$

Thus $w^{*}$ is the solution of the Stokes equation (1.4) with

$$
\left(\frac{d w^{*}}{d \tilde{x}}\right)=\tilde{M}^{*}, \quad \text { at } \tilde{x}=1 .
$$


The explicit formula for $w^{*}$ may be obtained directly by replacing $A$ and $B$ by 0 and $\tilde{M}^{*}$ respectively in (1.6). However the integral representation $(4.4 \mathrm{~b})$ will be useful.

\subsection{Asymptotic formulas for all real values of $\boldsymbol{k}$.}

THEOREM 4.1.

$$
\begin{gathered}
k>1 \Rightarrow \varepsilon M^{*}=O(1), \\
k=1 \Rightarrow \varepsilon M^{*}=O(\nu(\varepsilon)), \quad \nu(\varepsilon)=|\ln \varepsilon|^{-1}, \\
k<1 \Rightarrow \varepsilon^{k} M^{*}=O(1) .
\end{gathered}
$$

Proof. Let $x=O(1)$. Then $u^{*}(x)=O(1)$ and

$$
\begin{aligned}
u^{*}<1 \Rightarrow u^{*}>T^{*}[1] & =\varepsilon^{k} M^{*} \int_{\varepsilon}^{x} s^{-k} e^{-\beta-(s-\varepsilon)} d s \\
& =\varepsilon^{k} M^{*} e^{-\beta+\varepsilon}\left[E_{k}(\varepsilon)-E_{k}(x)\right] .
\end{aligned}
$$

For $k \neq 1$,

$$
0<u^{*}<T^{*}[0]=\varepsilon^{k} M^{*} \frac{\left[x^{1-k}-\varepsilon^{1-k}\right]}{1-k}
$$

For $k=1$,

$$
0<u^{*}<\varepsilon M^{*}[\ln (x)-\ln (\varepsilon)] .
$$

Now let $\varepsilon \downarrow 0$. For $k \geqq 1, E_{k}(\varepsilon)$ dominates the bracket in (a) and is $O\left(\varepsilon^{-k+1}\right)$ for $k>1$ and $O(|\ln \varepsilon|)$ for $k=1$. Thus (a) implies $\varepsilon M^{*} \leq 1$ and (b) implies $\varepsilon M^{*} \geq 1$ for $k>1$. This proves (4.5a). Similarly, for $k=1$, from (a) and (c)

$$
\varepsilon M^{*}|\ln \varepsilon| \leq 1, \quad \varepsilon M|\ln \varepsilon| \geq 1
$$

which proves (4.5b). Finally, if $k<1$

$$
E_{k}(\varepsilon)=O(1)
$$

so that (a) and (b) prove (4.5c).

Comments. For $k>1, M^{*}$ is of order $\varepsilon^{-1}$, independent of $k$, and in the limiting case $k=1$ its order is $\nu(\varepsilon) \varepsilon^{-1}$. Comparison with (1.14) shows that these are exactly the orders of $M_{0}=$ first approximation to $M^{*}$. Since subsequent terms of the expansion of $M^{*}$ have higher order, $(4.5 \mathrm{a})$ and $(4.5 \mathrm{~b})$ thus agree with a result of singular perturbation techniques. ${ }^{7}$ The result $(4.1 \mathrm{c})$ indicates that the same techniques are not applicable for $k<1$. This was already verified for $k=0$ and will be confirmed by the next theorem.

THEOREM 4.2. If $\varepsilon \downarrow 0$ then $u^{*}(x) \uparrow 1$ uniformly in maximal order domains depending on $k$,

$$
\begin{array}{ll}
k>1: x \geqq \eta, & \eta>\varepsilon, \\
k=1: x \geqq \eta, & |\ln \eta|^{-1}>\nu(\varepsilon), \\
k<1: x \geqq \eta, & \eta>1 .
\end{array}
$$

${ }^{7}$ This is, however, a weak statement. Stronger statements about the values of $M^{*}-M_{0}$ will be proved later (Theorem 4.4). 
Proof. Define

$$
I_{x_{1}}^{x_{2}}=\int_{x_{1}}^{x_{2}} s^{-k} \exp \left[-\beta u^{*}(s)-U^{*}(s)\right] d s .
$$

For $k>1$. From (4.5a) one finds

$$
1-u^{*}(\eta)=\varepsilon^{k} M^{*} I_{\eta}^{\infty}<\varepsilon^{k} M^{*} \int_{\eta}^{\infty} s^{-k} d s=\frac{\varepsilon^{k} M^{*} \eta^{k-1}}{k-1} \frac{\cup}{\cap}\left(\frac{\varepsilon}{\eta}\right)^{k-1} .
$$

Thus the domain of validity of 1 as an approximation to $u^{*}$ is at least as large as given by (4.6a). However, it could not be any larger since $u^{*}(\varepsilon)=0$.

For $k=1$. Write

$$
I_{\eta}^{\infty}=I_{n}^{\bar{x}}+I_{\bar{x}}^{\infty}, \quad \bar{x} \text { fixed }(\bar{x}=o(1))
$$

Then

$$
\exp \left[-\beta u^{*}(\bar{x})-U^{*}(\bar{x})\right] \int_{\eta}^{\bar{x}} s^{-1} d s<I_{\eta}^{\bar{x}}<\int_{\eta}^{\bar{x}} s^{-1} d s=\ln \frac{\bar{x}}{\eta} .
$$

Since the factor multiplying the first integral in (d) is $O(1)$,

$$
I_{\eta}^{\bar{x}} \frac{\cup}{\cap}|\ln \eta| \text {. }
$$

Furthermore

$$
I_{x}^{\infty} \stackrel{\cup}{\cap} 1
$$

Thus

$$
|\ln \eta|^{-1}>\nu(\varepsilon) \Rightarrow \varepsilon M^{*} I_{\eta}^{\infty} \rightarrow 0 \text {. }
$$

Since by (e) and (4.5b), $\varepsilon M^{*} I_{\eta}^{\bar{x}}$ does not tend to zero if $|\ln \eta|^{-1} \cup \frac{\cup}{\cap} \nu$ ), the proof of (4.6b) is complete.

For $k<1$. From (4.5c) one finds that $\varepsilon^{k} M^{*} I_{\eta}^{\infty}$ tends to zero only if the integral itself tends to zero which is equivalent to $\eta \uparrow \infty$ or $\eta>1$.

Comments. 1) The proof contains statements which actually are somewhat stronger than the theorem itself. For instance, (b) gives an upper bound for the order of $1-u^{*}(\eta)$ for $k>1$ etc.

2) The theorem verifies asymptotic results previously obtained by nonrigorous arguments in the literature cited. For instance, it had been assumed on intuitive grounds that for $k \geqq 1$ the outer limit of $u^{*}(x)$ was unity. This statement appears here in the form $\eta \cup \cup \cup 1 \Rightarrow u^{*}(\eta) \rightarrow 0$. Since $u^{*}(x)$ is monotone increasing with $x$ to $u(\infty)=1$ it follows that the same is true for $n>1$. This is obvious anyway since $\eta>1$ implies $x \rightarrow \infty$. The estimates of $1-u^{*}(\eta)$ for $\eta<1$ follow if one assumes that $g_{0}(\tilde{x})$, as given by (1.14), is an approximation to $u^{*}$ valid to order unity in this order range. Only (4.6c) contains a new result. It was known from the explicit formulas for $u^{*}$ in the case $k=0$. It has now been shown for all $k>1$. Thus the techniques used for $k \geqq 1$ are not applicable for $k<1$; it is questionable whether any form of matched asymptotic expansions can be used for the latter case.

LEMMA 4.1.

$$
\varepsilon<x<\infty \Rightarrow u^{*}(x)<w^{*}(x) .
$$

Proof. The lemma is trivial for $\beta=0$ since in that case $w^{*}=u_{1}^{*}=T^{*}\left[u_{0}\right], u_{0}=0$. For $\beta=1$ we note that at $x=\varepsilon, w^{*}=u^{*}, d w^{*} / d x=d u^{*} / d x, d^{2} w^{*} / d x^{2}=d^{2} u^{*} / d x^{2}$, 
$d^{3} w / d x^{3}>d^{3} u^{*} / d x^{3}$. Hence there exists a maximal open interval $\left(\varepsilon, x_{1}\right)$ on which $u^{*}(x)<w^{*}(x)$. Assume $x_{1} \neq \infty$. Then, at $x=x_{1}, u^{*}\left(x_{1}\right)=w^{*}\left(x_{1}\right)$ and $d u^{*} / d x \geqq$ $d w^{*} / d x$. On the other hand,

$$
\left(\frac{d w^{*}}{d x}\right)_{x=x_{1}}=\varepsilon^{k} M^{*} x_{1}^{-k} e^{-w^{*}\left(x_{1}\right)}
$$

and

$$
\left(\frac{d u^{*}}{d x}\right)_{x=x_{1}}=\varepsilon^{k} M^{*} x_{1}^{-k} e^{-w^{*}\left(x_{1}\right)}-U^{*}\left(x_{1}\right)<\left(\frac{d w}{d x}\right)_{x=x_{1}}
$$

This proves the lemma by contradiction.

\subsection{Asymptotic formulas for $k \geqq 1$.}

THEOREM 4.3.

$$
\begin{aligned}
& k>1 \text { and } k \neq 2 \Rightarrow w^{*}(x)-u^{*}(x) \leq \varepsilon, \quad x \text { in }[\varepsilon, \infty], \\
& k=2 \Rightarrow w^{*}(x)-u^{*}(x) \leq \varepsilon|\ln \varepsilon|, \quad x \text { in }[\varepsilon, \infty], \\
& k=1 \Rightarrow w^{*}(x)-u^{*}(x) \leq \nu(\varepsilon), \quad x \text { in }[\varepsilon, \bar{x}], \quad \bar{x}=O(1)
\end{aligned}
$$

and

$$
w^{*}(x)-u^{*}(x)<1 \quad \text { when } x>1 \text { and } \nu(\varepsilon)<|\ln x|^{-1} \text {. }
$$

Proof.

(a)

$$
\begin{aligned}
w^{*}(x)-u^{*}(x) & =\varepsilon^{k} M^{*} \int_{\varepsilon}^{x} s^{-k}\left[e^{-\beta w^{*}(x)}-e^{-\beta u^{*}(s)-U^{*}(s)}\right] d s \\
& \leqq \varepsilon^{k} M^{*} \int_{\varepsilon}^{x} s^{-k} e^{-u^{*}(s)}\left[1-e^{-U^{*}(s)}\right] \\
& \leqq \int_{\varepsilon}^{x} s^{-k}\left[1-e^{-U^{*}(s)}\right] d s \\
& \leqq \varepsilon^{k} M^{*} \int_{\varepsilon}^{x} s^{-k}\left(1-e^{-s}\right) d s .
\end{aligned}
$$

For $k>1$ and $k \neq 2$,

(b)

$$
\int_{\varepsilon}^{x} s^{-k}\left(1-e^{-s}\right)=O\left(\varepsilon^{-k+2}\right)
$$

Thus (4.8a) follows from (a), (b) and (4.5a).

For $k=2$,

(c)

$$
\left.\int_{\varepsilon}^{x} s^{-2}\left(1-e^{-s}\right)=O|\ln \varepsilon|\right) .
$$

Thus (4.8b) follows from (a), (c) and (4.5a).

For $k=1$,

(d)

$$
\int_{\varepsilon}^{x} s^{-1}\left(1-e^{-s}\right) d s=\ln x-\ln \varepsilon-E_{1}(\varepsilon)+I_{1}(x) .
$$


Since for small values of $t$

$$
E_{1}(t)=-\ln t-\gamma+O(t)
$$

it follows that $-\ln \varepsilon-E_{1}(\varepsilon)=O(1)$. Similarly $\ln (x)+E_{1}(x)=o(1)$ for $x<1$. Thus

$$
x \leqq 1 \Rightarrow w^{*}(x)-u^{*}(x) \leq \nu(\varepsilon) .
$$

For $x>1$, the term $\ln x$ dominates in (d). This concludes the proof of (4.8c).

Comments. The complications for $k=1$ arise as usual from the fact that the indefinite integral of $s^{-1}$, namely $\ln x$, is large in absolute value both for $x$ large and $x$ small. The special estimate for $k=2$ is consistent with singular perturbation theory. In this case the first correction to $g_{0}(\tilde{x})$ has a term of order $\varepsilon$ and a term of order $|\ln \varepsilon|$.

THEOREM 4.4.

$$
\frac{M^{*}}{M_{0}}=1+o(1)
$$

Proof. The functions $w^{*}$ and $g_{0}$ have three things in common: 1) they satisfy the same equation, 2) their value at $x=\varepsilon$ is zero, and 3) they match with $u^{*}$ in some domain. This third condition follows for $g_{0}$ by construction and for $w^{*}$ from Theorem 4.3.

A consequence of the first two conditions is that $g_{0}$ and $w^{*}$ have the forms given by (1.6) with $A=0$ whereas $B$ will have to be determined by matching. As may easily be seen matching by requiring the outer limit of the inner solution to be unity gives the same result as a more orthodox choice of the overlap domain. To fix the ideas, let $\beta=0$ and $k>1$. Putting $A=0$ in (1.6b) and assuming its outer limit to be equal to 1 gives (cf. (1.14b))

$$
\lim B=k-1
$$

Thus

$$
\lim \tilde{M}^{*}=\tilde{M}_{0}=k-1
$$

which proves the theorem for this special case. More generally, all functions given by (1.6) are continuous in $B$ which proves Theorem 4.4 for all special cases.

Corollary.

The domain of validity of the approximation $g_{0}(\tilde{x})$ to order unity may be inferred from the domain of validity of $w^{*}$ as given by Theorem 4.3 .

Comments. A general principle for proving the validity of an inner expansion. It seems worthwhile to extract the essentials of the proof of (4.10). The statements given below may of course be generalized further.

Assume that we have a two-point boundary value problem for the interval $\left[x_{1}, x_{2}\right]$ and that there is a boundary layer at $x_{1}$. Assume further that this problem has a unique solution $u^{*}$, at least for $\varepsilon$ sufficiently small. Then $u^{*}\left(x_{1}\right)=A^{*}$ and $\left(d u^{*} / d x\right)_{x=x_{1}}=B^{*}$ are determined, at least in principle (for instance $B^{*}$ may not be known exactly), and the problem may be rephrased as an initial-value problem. Such problems are often much easier to study than a boundary value problem. Let $w^{*}$ be the exact solution of the boundary layer equation with the initial conditions $w^{*}\left(x_{1}\right)=A^{*},\left(d w^{*} / d x\right)_{x=x_{1}}=$ $B^{*}$, and $g_{0}$ the boundary layer solution obtained by matching, giving a value $B_{0}$ for $\left(d g_{0} / d x\right)_{x=x_{1}}$. Assume that one can prove that $\left|u^{*}-w^{*}\right|=o(1)$ for a region in which matching is possible. If the solution of the boundary layer equation depends 
continuously on one parameter $B$ (= the derivative at $\left.x=x_{1}\right)$ it then follows that $\left|u^{*}-g_{0}\right|=o(1)$ for all $x$ for which $\left|u^{*}-w^{*}\right|=o(1)$.

In the present case the estimate of $u^{*}-w^{*}$ depended on the fact that the initial value problem for both functions could be rephrased as an integral equation. If $u^{*}-w^{*}$ can be evaluated by other methods, the principle announced above is not only valid but also useful. Note that strictly speaking matching does not determine $B=B_{0}$ but rather $B=B_{0}+o\left(B_{0}\right)$. This fact was emphasized, and used, in Kaplun (1957, p. 597).

THEOREM 4.5 .

$$
\left|u^{*}(x)-w^{*}(x)\right|=o(1) \Rightarrow\left|u^{*}(x)-g_{0}\left(x \varepsilon^{-1}\right)\right|=o(1) .
$$

Proof. This statement is obvious and discussed in the comments given above. Thus from Theorem 4.3 we know the validity to order unity of the inner approximation $g_{0}$.

The validity of the leading terms of the outer and inner expansions have now been established. We shall give an example of how one may treat higher-order approximations. For we take $\beta=1$ and $k=1$.

TheOREM 4.6. For $k=1, \beta=1$,

$$
\frac{u^{*}-\left[1-((e-1) / e) \nu(\varepsilon) E_{1}(x)\right]}{\nu(\varepsilon)} \rightarrow 0
$$

for $x$ fixed. ${ }^{8}$

Proofs.

$$
\nu(\varepsilon)^{-1}\left(1-u^{*}\right)=\frac{\varepsilon M^{*}}{\nu(\varepsilon)} \int_{x}^{\infty} s^{-1} \exp \left[-u^{*}(s)-U^{*}(s)\right] d s
$$

Taking the limit as $\varepsilon \downarrow 0$ we find from (4.9) and, (1.14c)

$$
\frac{\varepsilon M^{*}}{\nu(\varepsilon)} \rightarrow(e-1) \text {. }
$$

Furthermore, from (4.6b) for $x=O(1)$,

$$
u^{*}(s) \rightarrow 1, \quad U^{*}(s) \rightarrow s .
$$

Thus the limit of (a) is

$$
(e-1) \int_{x}^{\infty} s^{-1} e^{-1-s} d s=\frac{e-1}{e} E_{1}(x)
$$

This proves (4.12).

Comments. The values $k=1$ and $\beta=1$ were chosen for concreteness. The method of proof works equally well for any $k \geqq 1, \beta=0$ or $\beta=1$, if one uses the first two terms of the outer expansion as given by (1.14). We may rephrase Theorem 4.6 by stating that the first two terms of the outer expansion are valid to order $\nu(\varepsilon)$ in the domain $[\eta, \infty], \eta=O(1)$. By Kaplun's extension theorem this domain may be enlarged slightly; there is an $\eta_{0}<1$, such that the domain of validity to the same order is $[\eta, \infty]$ for any $\eta>\eta_{0}$. It is known that there is a trade-off between order of validity and domain of validity. If an approximation is valid to order $\zeta(\varepsilon)$ in a domain $[\eta(\varepsilon), 1]$, then the domain of validity is sometimes extended ( $\eta$ replaced by $\left.\eta^{*}<\eta\right)$ if

\footnotetext{
${ }^{8}$ Note that the bracket represents the first two terms of the outer expansion as determined by matched asymptotic expansions; see (1.15c).
} 
$\zeta$ is replaced by $\zeta^{*}, \zeta^{*}>\zeta$. This might be studied, for $k>1$, with the aid of the estimate of the order of $1-u^{*}(\eta)$ given by (b) in the proof of Theorem 4.2 ; however, it will not be discussed here. We shall also omit discussion of higher-order approximations; the general method given here should work for any partial sum of inner and outer expansions. A fair sample of proofs for partial expansions is sufficient. After that the interesting theorems would be those for expansions containing infinitely many terms. We shall formulate one such problem.

Problem. Let $k=1$ and $\beta=1$ (similar statements for $\beta=0$ are obvious). The inner expansion need not be expressed as a partial sum. A neater form ${ }^{9}$ is to express it as a sequence $g^{(0)}, g^{(1)}$ etc., where

$$
g^{(m)}=\ln \left[1+\left(\sum_{j=0}^{m} D_{j} \nu^{j+1}\right) \ln \tilde{x}\right], \quad D_{j}=\text { constant }
$$

For instance,

$$
\begin{aligned}
g^{(2)} & =\ln \left[1+D^{(2)}(\varepsilon) \ln \tilde{x}\right], \\
D^{(2)} & =(e-1)\left[(\nu+\gamma) \nu^{2}+\gamma^{2}\left(1-\frac{e-1}{e} 2 \ln 2\right) \nu^{3}\right] .
\end{aligned}
$$

Assume now that a function $D^{(\infty)}(\varepsilon)$ exists, to which the formal sum $\sum_{j=0}^{\infty} D_{j} \nu^{j+1}$ is asymptotic (it is of course not unique). Put

$$
g^{(\infty)}(\tilde{x})=\ln \left[1+D^{(\infty)}(\varepsilon) \ln \tilde{x}\right]
$$

One may then pose the problem whether

$$
\nu^{-m}\left[u^{*}-g^{(\infty)}(\tilde{x})\right] \rightarrow 0, \quad \text { any } m>0,
$$

for

$$
\varepsilon<x \leq \varepsilon^{b}, \text { any } b \text { in }(0,1] .
$$

(As is well-known $g^{(\infty)}$ does not represent the complete inner expansion; the term $u(d u / d x)=\varepsilon u(d u / d \tilde{x})$ has been neglected since $\varepsilon$ is transcendentally small with respect to $\nu(\varepsilon)$.)

\section{REFERENCES}

WM. B. BUSH (1971), On the Lagerstrom mathematical model for viscous flow at low Reynolds numbers, this Journal, 20, pp. 279-287.

J. D. Cole (1968), Perturbation Methods in Applied Mathematics, Blaisdell, Waltham, MA.

Ph. HARTMAN (1964), Ordinary Differential Equations, John Wiley, New York.

G. C. HsIAO (1973), Singular perturbations for a nonlinear differential equation with a small parameter, SIAM J. Math. Anal., 4, pp. 283-301.

S. KAPLUN (1957), Low Reynolds number flow past a circular cylinder, J. Math. Mech., 6, pp. 595-603. Reprinted in Kaplun (1967).

(1967), Fluid Mechanics and Singular Perturbations, P. A. Lagerstrom, L. N. Howard and C. S. Liu, eds., Academic Press, New York.

S. KAPlun AND P. A. LAGerstrom (1957), Asymptotic expansions of Navier-Stokes solutions for small Reynolds numbers, J. Math. Mech., 6, pp. 585-593. Reprinted in Kaplun (1967).

P. A. LAGERSTROM (1961), Méthodes asymptotiques pout l'étude des équations de Navier-Stokes, Lecture notes, Institut Henri Poincaré, Paris; English transl. by T. J. Tyson, California Institute of Tech. Pasadena, CA, 1965.

\footnotetext{
${ }^{9}$ The coefficient of $\ln \tilde{x}$ in $(4.13 a)$ is $\left(d g^{(m)} / d \tilde{x}\right)_{\tilde{x}=1}$.
} 
(1976), Forms of singular asymptotic expansions in layer-type problems, Rocky Mountain J. Math. 6 , No. 4, pp. 609-635.

P. A. LAgERSTROM AND R. G. CASTEN (1972), Basic concepts underlying singular perturbation techniques, SIAM Rev., 14, pp. 63-120.

P. A. LAgerstrom AND J. D. COle (1955), Examples illustrating expansion procedures for the NavierStokes equations, J. Rational Mech. Anal., 4, pp. 817-882.

A. D. MACGillivray (1977), On a model of Lagerstrom, this Journal, to appear.

M. H. ProtTer AND H. F. Weinberger (1967), Maximum principles in differential equations, PrenticeHall, Englewood Cliffs, N.J.

I. Proudman AND J. R. A. PEARson (1957), Expansions at small Reynolds numbers for the flow past a sphere and a circular cylinder, J. Fluid Mech., 2, pp. 237-262.

H. WEYL (1942), On the differential equations of the simplest boundary-layer problems, Ann. of Math. 43, pp. 381-407. Reprinted in Weyl (1956).

- (1956), Selecta Hermann Weyl, Birkhäuser Verlag, Basel and Stuttgart. 